\title{
Autoantibodies to Osteoprotegerin are Associated with Low Hip Bone Mineral Density and History of Fractures in Axial Spondyloarthritis: A Cross-Sectional Observational Study
}

\author{
Barbara Hauser $^{1}$ - Sizheng Zhao ${ }^{2,3}$ • Micaela R. Visconti ${ }^{1}$ Philip L. Riches ${ }^{1}$. \\ William D. Fraser ${ }^{4} \cdot$ Isabelle Piec $^{4} \cdot$ Nicola J. Goodson ${ }^{2,3} \cdot$ Stuart H. Ralston $^{1}$
}

Received: 14 December 2016/Accepted: 12 May 2017/Published online: 22 May 2017

(c) The Author(s) 2017. This article is an open access publication

\begin{abstract}
Osteoporosis is a recognised complication of axial spondyloarthritis (axSpA) and is thought to be due to functional impairment and the osteoclast-activating effects of proinflammatory cytokines. The development of autoantibodies to OPG (OPG-Ab) has been associated with severe osteoporosis and increased bone resorption in rheumatoid arthritis. In this study, we screened for the presence of OPG-Ab in axSpA and reviewed their clinical significance. We studied 134 patients, recruited from two centres in the United Kingdom. Their mean age was 47.5 years and $75 \%$ were male. Concentrations of OPG-Ab were related to bone mineral density (BMD) and fracture history using linear and logistic regression models adjusting for age, gender, disease duration and activity, body mass index and bisphosphonate use. We detected OPG-Ab in 11/134 patients (8.2\%). Femoral neck and total hip BMD were significantly reduced in OPG-Ab positive patients ( 0.827 vs. $0.967 \mathrm{~g} / \mathrm{cm}^{2}, p=0.008$ and 0.868 vs. $1.028 \mathrm{~g} /$ $\mathrm{cm}^{2}, p=0.002$, respectively). Regression analysis showed that the presence of OPG-Ab was independently associated
\end{abstract}

Barbara Hauser and Sizheng Zhao are joint first authors in alphabetical order.

Nicola J. Goodson

ngoodson@liverpool.ac.uk

1 Centre for Genomics and Experimental Medicine, Institute of Genetics and Molecular Medicine, University of Edinburgh, Edinburgh, UK

2 Department of Academic Rheumatology, Aintree University Hospital, Liverpool L9 7AL, UK

3 Musculoskeletal Biology I, Institute of Ageing and Chronic Disease, University of Liverpool, Liverpool, UK

4 Department of Medicine, University of East Anglia, Norwich, UK with total hip osteopenia $\left(\mathrm{OR}_{\mathrm{adj}} 24.2 ; 95 \%\right.$ CI $\left.2.57,228\right)$ and history of fractures $\left(\mathrm{OR}_{\mathrm{adj}} 10.5 ; 95 \% \mathrm{CI} 2.07,53.3\right)$. OPG-Ab concentration was associated with total hip BMD in $\mathrm{g} / \mathrm{cm}^{2}(\beta=-1.15 ; 95 \% \mathrm{CI}-0.25,-0.04)$. There were no associations between OPG-Ab concentration and bone turnover markers, but free sRANKL concentrations were lower in OPG-Ab-positive patients (median 0.04 vs. $0.11 \mathrm{pmol} / \mathrm{L}, p=0.050$ ). We conclude that $\mathrm{OPG}-\mathrm{Ab}$ are associated with hip BMD and fractures in axSpA suggesting that they may contribute to the pathogenesis of bone loss in some patients with this condition.

Keywords Ankylosing spondylitis · Axial spondyloarthritis · Osteoporosis - Bone mineral density · Osteoprotegerin

\section{Introduction}

Axial spondyloarthropathy (axSpA) is characterised by inflammation of the sacroiliac joints and entheses. Disease progression involves not only new bone formation at sites of inflammation but also, paradoxically, an increased risk of osteoporosis and vertebral fractures [1-3]. Osteoporosis is present in up to $25 \%$ of patients [4] and vertebral fractures have been reported in up to $30 \%$ of patients $[5,6]$. Both can occur in early disease [7, 8]. In contrast to the general population, axSpA-associated osteoporosis is more prevalent in men and occurs at younger ages [8], which limits the value of conventional fracture risk assessment tools such as FRAX $^{\circledR}[9]$.

AxSpA shares many factors in common with other inflammatory arthropathies that are associated with accelerated bone loss, including impaired physical mobility [8] and increased production of cytokines such as TNF and 
IL17 which stimulate osteoclastic bone resorption [10]. Although the patterns of bone involvement differ in rheumatoid arthritis (RA) and axSpA, the final common pathway of bone loss in both cases is increased osteoclastic bone resorption which is critically dependent on the stimulatory effects of RANKL, counterbalanced by the inhibitory effects of OPG [11]. However, axSpA also differs in that it has younger age of onset, male predominance and lower systemic inflammatory burden. Other novel factors may therefore be associated with the development of premature osteoporosis and fractures in the axSpA population.

Autoantibodies to osteoprotegerin (OPG-Ab) were previously identified as a cause of high turnover osteoporosis in a patient with coeliac and autoimmune thyroid disease [12]. OPG-Abs have also been detected in patients with RA, where they were associated with increased bone resorption [13] and in coeliac disease where they were associated with reduced BMD [14]. The aim of this study was to determine if OPG-Abs were present in axSpA and to explore whether they were associated with BMD or history of fractures.

\section{Methods}

We enrolled consecutive axSpA patients who presented to specialist rheumatology clinics at Aintree University Hospital in Liverpool and the Western General Hospital in Edinburgh, between July 2011 and February 2015. Patients were included in the study if they met the modified New York criteria for ankylosing spondylitis [15] and/or the ASAS criteria for axSpA [16].

We recorded demographic information, disease characteristics (age at diagnosis, HLA-B27 status if available, extra-axial involvement), and current medication [NSAIDs, DMARDs, calcium/vitamin D, bisphosphonates and TNF inhibitors (TNFi)], ever-smoking, current glucocorticoid use and body mass index (BMI). We enquired about a history of clinical fractures and fracture site (vertebral or non-vertebral). Clinical notes and radiographs were reviewed to verify self-reported fractures. Disease duration was defined as the number of years since first diagnosis. Disease activity was assessed using the Bath Ankylosing Spondylitis Disease Activity Index (BASDAI), spinal pain visual analogue scale (spVAS) and Bath AS Functional Index (BASFI). Blood samples were obtained for erythrocyte sedimentation rate (ESR) and C-reactive protein (CRP) using the local hospital laboratories. Serum samples were also prepared at the time of enrolment and stored at $-80{ }^{\circ} \mathrm{C}$ until use for measurement of specialised markers of bone turnover and OPG-Ab as described below.

Bone mineral density (BMD) was assessed using anteroposterior DXA of the lumbar spine (L1-L4), femoral neck and total hip using Lunar iDXA (GE Healthcare) at Liverpool and Hologic QDR4500 at Edinburgh. Hologic BMD values in $\mathrm{g} / \mathrm{cm}^{2}$ were converted to Lunar equivalents as manufacturer's recommendations [17, 18]. Osteopenia and osteoporosis were defined as $T$-scores $<-1$ and $\leq-2.5$, respectively [19].

Measurement of OPG-Ab was performed at the Centre for Genomic and Experimental Medicine by an indirect ELISA developed in-house as described previously [14]. A standard curve comprising serial dilutions of serum from the index case was run on each plate with an arbitrary value of 100 units assigned to neat serum. The concentrations of OPG-Ab were estimated from this standard curve. Each sample was run in triplicate and repeated at least once on a different day. One result of each triplicate was discarded as an outlier if it was more than three standard deviations outside the mean of two most concordant values, except where the two most concordant values were identical. The mean of the remaining values was used for analysis. Interand intra-assay coefficients of variation $(\mathrm{CV})$ for positive OPG-Ab ELISA titre were 16.6 and $17.3 \%$, respectively. A previous study of 100 healthy controls [mean age 57.8 $(\mathrm{SD} \pm 12)]$ with normal BMD demonstrated a mean titre of $3.46(\mathrm{SD} \pm 3.64)$ and was used to define the limit for OPG-Ab positivity [14]. Positive OPG-Ab was defined as titres three standard deviations above the mean [20], which equates to $\geq 14.3$ units.

Measurements of OPG, free soluble RANKL (sRANKL), the N-terminal pro-peptide of type I collagen (PINP) and the C-terminal type 1 crosslinked telopeptide (CTX) were performed at the University of East Anglia bioanalytical facility. Measurements of CTX $(\mathrm{ng} / \mathrm{ml})$ and PINP (ng/ml) were performed on COBAS 6000 (Roche Diagnostics) with an inter-assay $\mathrm{CV}$ of 3.9 and $4.0 \%$, respectively. Concentrations of OPG ( $\mathrm{pmol} / \mathrm{L})$ and free sRANKL (pmol/L) were measured using ELISA kits (Biomedica, Austria) following manufacturer's instructions. Performance of these kits (on duplicated samples) was intra-assay CV $3.1 \%$ for OPG and $3.8 \%$ for sRANKL. Inter-assay CV were $6.6 \%$ for OPG $(n=4)$ and $4.1 \%$ for sRANKL $(n=3)$ across the standard curve concentrations for the assay. Reference ranges for sRANKL, CTX and PINP were obtained from the manufacturer literature, and for OPG from the population study [21]: OPG (male 0.124-10.690 pmol/L; female $\quad 0-11.475 \mathrm{pmol} / \mathrm{L}$ ), sRANKL (male $0.022-0.381 \mathrm{pmol} / \mathrm{L}$; female 0.020-0.329 pmol/L), CTX (male $0.158-0.442 \mathrm{ng} / \mathrm{ml}$; female $0.092-0.436 \mathrm{ng} / \mathrm{ml}$ ) and PINP (male $20-76 \mathrm{ng} / \mathrm{ml}$; female $15-59 \mathrm{ng} / \mathrm{ml}$ ).

Statistical analyses were performed using Stata13. Data from both study sites were combined for analyses. Comparative analysis was performed using Mann-Whitney $U$ test for non-normally distributed variables, Student's 
$t$ test for normally distributed variables and Fisher's exact test for categorical variables. Associations between OPGAb concentration and OPG, free sRANKL, CTX and PINP were assessed using Spearman's rank correlation in the total cohort and separately in patients who were not receiving bisphosphonates. Association between positive OPG-Ab and disease characteristics was assessed with univariate logistic regression. Correlation between BMD $\left(\mathrm{g} / \mathrm{cm}^{2}\right)$ and OPG-Ab concentration was demonstrated using scatter graphs.

In the first instance, regression models were used to assess associations between measures of bone density and OPG-Ab positivity as the independent variable. Linear models were used for BMD $\left(\mathrm{g} / \mathrm{cm}^{2}\right), T$ - and Z-scores as dependent variables, adjusted for age (not included in $Z$ score model), gender, disease duration, bisphosphonate use, BASDAI and BMI. Logistic models were used for osteopenia, osteoporosis and fractures as dependent variables, using the same covariates. No imputation was performed for missing data; therefore, multivariable models were limited to a subgroup with complete data for all covariates. In addition to analyses of OPG-Ab positivity, the same linear and logistic models were generated using OPG-Ab concentration as the independent variable. Residuals of linear models were tested against a normal distribution using kernel density plots and skewness-kurtosis test. Finally, backward stepwise regression analyses were performed to identify independent explanatory variables for total hip, femoral neck and spine $\mathrm{g} / \mathrm{cm}^{2}$ each as the dependent variable, using $p>0.1$ as criteria for removal from the full model. Significant association was defined as $p<0.05$.

\section{Results}

The axSpA cohort comprised 134 patients: 53 from Edinburgh and 81 from Liverpool. There were no significant differences in patient or disease characteristics between the two study sites (data not shown). The median OPG-Ab titre was 2.8 (range 0.2, 36; interquartile range [IQR] 1.4, 4.3). A positive OPG-Ab result was detected in 11 patients $(8.2 \%)$. There was no difference in proportion of positive OPG-Ab between study sites $(p=0.526)$. Demographics and disease characteristics of patients positive and negative for OPG-Ab are shown in Table 1 . The cohort was predominantly male $(75 \%)$ with a mean age of 47.5 years $(\mathrm{SD} \pm 15)$ and medium disease duration of 6.4 years [IQR 1.9, 17.8]. Patients positive for OPG-Ab were older and had longer disease duration. They were also lower in height, although BMI was similar between the groups.

The BASDAI scores were generally high (median 5.4) with no significant difference between the OPG-Ab positive and negative groups. Peripheral joint involvement was reported in $35 \%$ of patients and $11 \%$ had associated inflammatory bowel disease. A third of patients were treated with TNFi and 64\% were on NSAIDs at the time of recruitment. A greater proportion of patients positive for OPG-Ab were currently using TNFi $(p=0.043)$ and bisphosphonates $(p=0.006)$.

Bone density measurements were available in 120 patients, measured within a median of 0.2 months [IQR $-9.6,2.9]$ from the study visit. Existing DXA results were available for $50 \%$ of patients and a further $50 \%$ had scans organised at the time of the study assessment. Most scans (79\%) were performed within 12 months of the study assessment. Spinal BMD was not measured for one patient due to significant aortic calcification. Hip BMD was not measured in two patients who had bilateral hip replacements. Differences between patients positive and negative for OPG-Ab are shown in Table 1. No significant differences in BMD were observed at the spine. Total hip and femoral neck BMD $\left(\mathrm{g} / \mathrm{cm}^{2}\right)$ and T-scores were all significantly lower in OPG-Ab positive patients. The same was true for Z-score (data not shown). Osteopenia of the total hip and femoral neck was significantly more common in OPG-Ab-positive patients ( 82 vs. $28 \%, p<0.001$ and 82 vs. $35 \%, p=0.007$, respectively). The same was true for osteoporosis of total hip and femoral neck (18 vs. $1 \%$, $p=0.023$ and 27 vs. $6 \%, p=0.039$, respectively).

A history of fracture was reported by $35(26 \%)$ patients. Three patients (2\%) had multiple vertebral fractures and 32 (24\%) had non-vertebral fractures. OPG-Ab positive patients had a higher proportion of self-reported fractures $(p=0.007)$. Radiographs and/or clinical notes were available to confirm 17 of the self-reported fractures.

In the whole study cohort, the median level of OPG was $4.2 \mathrm{pmol} / \mathrm{L}$ [IQR 3.4, 4.9], sRANKL $0.11 \mathrm{pmol} / \mathrm{L}$ [IQR 0.04, 0.18], CTX $0.22 \mathrm{ng} / \mathrm{mL}$ [IQR 0.16, 0.32] and PINP $42 \mathrm{ng} / \mathrm{mL}$ [IQR 30, 55]. For OPG, 99\% of the cohort were within the reference range, $77 \%$ for sRANKL, $71 \%$ for CTX and $88 \%$ for PINP. Serum concentrations of OPG, sRANKL, PINP and CTX in relation to OPG-Ab status are shown in Fig. 1. Concentrations of free sRANKL were lower in OPG-Ab positive group ( $p=0.050$ ) but there was no significant difference between the groups for concentrations of OPG, NTX or PINP. An inverse correlation was observed between OPG-Ab concentration and total hip and femoral neck BMD but not for spine BMD (Fig. 2). Similar correlations were observed for $Z$-scores (not shown).

Univariate logistic regression demonstrated significant associations between OPG-Ab positivity and disease duration (OR 1.05; 95\% CI 1.01, 1.10) and age (OR 1.04; $95 \%$ CI 1.00, 1.09). Multi-adjusted regression models showed significant associations between positive OPG-Ab status and total hip BMD $\left(\mathrm{g} / \mathrm{cm}^{2}\right), T$ - and Z-scores 
Table 1 Patient and disease characteristics by OPG-Ab status

\begin{tabular}{|c|c|c|c|}
\hline & Positive $n=11$ & Negative $n=123$ & $p$-value \\
\hline \multicolumn{4}{|l|}{ Demographics } \\
\hline Age (years) & $56.1 \pm 15.5$ & $46.2 \pm 14.7$ & 0.036 \\
\hline Males & $8(73 \%)$ & $92(75 \%)$ & 1.000 \\
\hline HLA-B27 $(n=59)$ & $3(60 \%)$ & $39(72 \%)$ & 0.620 \\
\hline Age diagnosed & $43.3 \pm 15.1$ & $40.9 \pm 14.5$ & 0.610 \\
\hline Median years disease duration & $22.3[0.8,33.1]$ & $5.6[1.9,16.3]$ & 0.067 \\
\hline Ever smokers & $5(45 \%)$ & $40(33 \%)$ & 0.510 \\
\hline Height $(\mathrm{cm})(n=130)$ & $164 \pm 7.5$ & $171 \pm 9.6$ & 0.018 \\
\hline Weight $(\mathrm{kg})(n=132)$ & $78.6 \pm 14.5$ & $81.4 \pm 17.3$ & 0.609 \\
\hline BMI $\left(\mathrm{kg} / \mathrm{m}^{2}\right)(n=130)$ & $29.3 \pm 5.9$ & $27.8 \pm 5.8$ & 0.391 \\
\hline \multicolumn{4}{|l|}{ Disease activity } \\
\hline Median BASDAI $(n=132)$ & $3.9[2,5.7]$ & $5.5[3.2,7.7]$ & 0.137 \\
\hline Median spVAS $(n=126)$ & $5[2,8]$ & $6[2.9,8]$ & 0.741 \\
\hline Median BASFI $(n=106)$ & $4[3.4,8.5]$ & $5.3[3.4,7.4]$ & 0.869 \\
\hline Median ESR $(\mathrm{mm} / \mathrm{h})(n=111)$ & $14[5,27]$ & $8[5,17]$ & 0.420 \\
\hline Median CRP $(\mathrm{mg} / \mathrm{L})(n=109)$ & $1[1,6]$ & $4[1,9]$ & 0.230 \\
\hline \multicolumn{4}{|l|}{ Extra-axial involvement } \\
\hline Peripheral joint involvement & $4(36 \%)$ & $42(34 \%)$ & 1.000 \\
\hline Psoriasis & $2(18 \%)$ & $18(15 \%)$ & 0.671 \\
\hline Uveitis & $2(18 \%)$ & $44(36 \%)$ & 0.329 \\
\hline Inflammatory bowel disease & $1(9 \%)$ & $14(11 \%)$ & 1.000 \\
\hline \multicolumn{4}{|l|}{ Medication } \\
\hline TNF inhibitor & $7(64 \%)$ & $38(31 \%)$ & 0.043 \\
\hline Synthetic DMARD & 0 & $15(12 \%)$ & 0.256 \\
\hline NSAIDs & $6(55 \%)$ & $77(65 \%)$ & 0.520 \\
\hline Glucocorticoid & $1(9 \%)$ & $2(2 \%)$ & 0.228 \\
\hline Bisphosphonates & $4(36 \%)$ & $7(6 \%)$ & 0.006 \\
\hline Calcium and vitamin D & $7(64 \%)$ & $57(46 \%)$ & 0.271 \\
\hline \multicolumn{4}{|l|}{ Lumbar spine $^{\mathrm{a}}$} \\
\hline $\mathrm{g} / \mathrm{cm}^{2}$ & $1.226 \pm 0.189$ & $1.231 \pm 0.235$ & 0.947 \\
\hline$T$-score & $0.2 \pm 1.5$ & $0.1 \pm 2.0$ & 0.963 \\
\hline \multicolumn{4}{|l|}{ Femoral neck ${ }^{\mathrm{b}}$} \\
\hline $\mathrm{g} / \mathrm{cm}^{2}$ & $0.827 \pm 0.131$ & $0.967 \pm 0.166$ & 0.008 \\
\hline$T$-score & $-1.68 \pm 1.04$ & $-0.57 \pm 1.22$ & 0.004 \\
\hline Osteopenia & $9(82 \%)$ & $37(35 \%)$ & 0.007 \\
\hline Osteoporosis & $3(27 \%)$ & $6(6 \%)$ & 0.039 \\
\hline \multicolumn{4}{|l|}{ Total hip ${ }^{\mathrm{b}}$} \\
\hline $\mathrm{g} / \mathrm{cm}^{2}$ & $0.868 \pm 0.142$ & $1.028 \pm 0.157$ & 0.002 \\
\hline$T$-score & $-1.5 \pm 1.0$ & $-0.3 \pm 1.1$ & 0.001 \\
\hline Osteopenia & $9(82 \%)$ & $30(28 \%)$ & 0.001 \\
\hline Osteoporosis & $2(18 \%)$ & $1(1 \%)$ & 0.023 \\
\hline History of fractures & $7(64 \%)$ & $28(23 \%)$ & 0.007 \\
\hline
\end{tabular}

Data shown as mean \pm standard deviation; median [interquartile range]; number (percentage)

BASDAI Bath Ankylosing Spondylitis Disease Activity Index, spVAS spinal pain visual analogue scale, $B A S F I$ Bath AS Functional Index, $B M D$ bone mineral density, $O P G-A b$ anti-OPG antibody, $n$ number of available data)

${ }^{a}$ Measures of spine BMD were missing for one patient due to significant aortic calcification

b Measures of hip BMD were missing for two patients due to bilateral hip replacements 
Fig. 1 Biochemical markers of bone metabolism and OPG antibody status
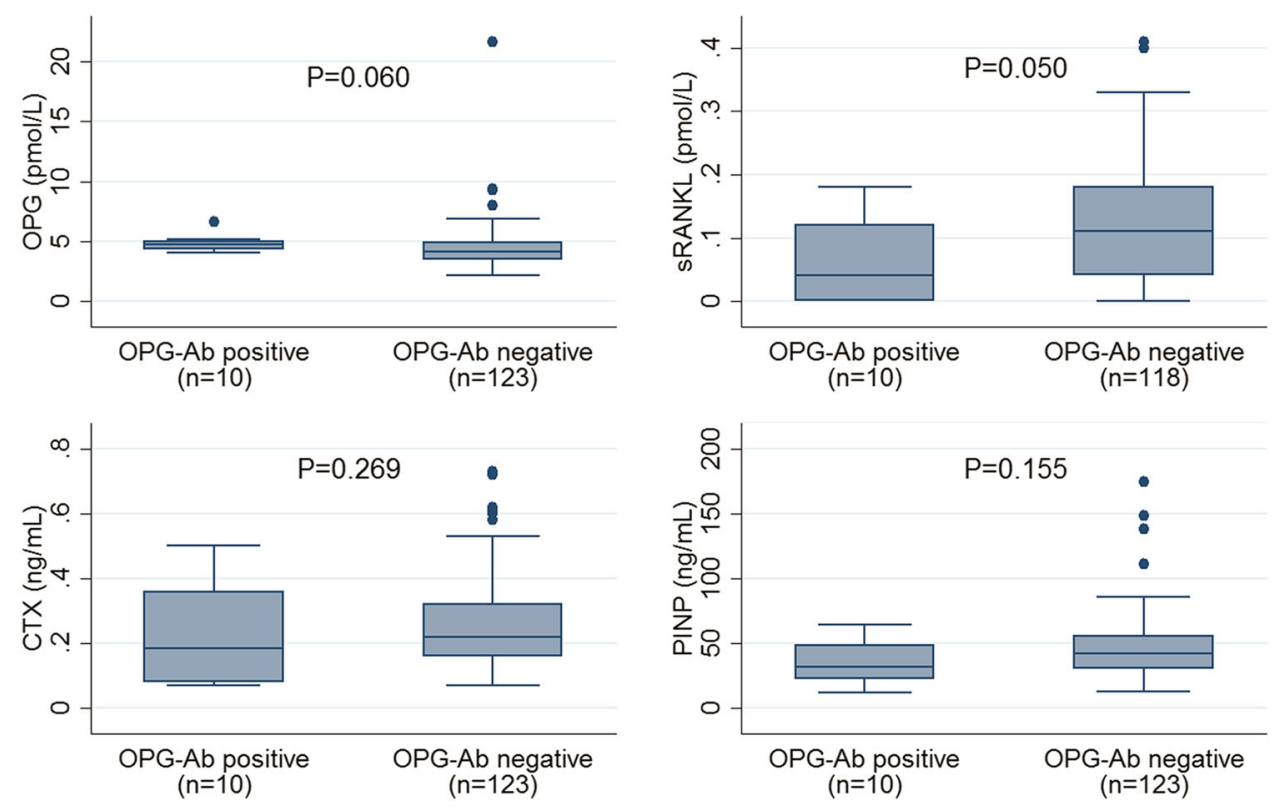

(Table 2). Similar trends were observed for femoral neck BMD but significance was reached only for the association between femoral neck Z-score and OPG-Ab positivity. Positive OPG-Ab status was strongly associated with osteopenia at the total hip $\left(\mathrm{OR}_{\mathrm{adj}} 24.2 ; 95 \% \mathrm{CI} 2.57,228\right)$ and femoral neck $\left(\mathrm{OR}_{\mathrm{adj}} 21.3 ; 95 \%\right.$ CI 1.96, 231) but not osteoporosis. In contrast, there was no association between OPG-Ab and lumbar spine BMD $\left(\mathrm{g} / \mathrm{cm}^{2}\right), T$ - or $Z$-scores. OPG-Ab positivity was significantly associated with history of self-reported fractures $\left(\mathrm{OR}_{\mathrm{adj}} 10.5\right.$; 95\% CI 2.07, 53.3). In regression models of OPG-Ab concentration, the same associations were demonstrated for measures of total hip BMD, osteopenia and previous fractures (Table 2). Post hoc analyses including TNFi as additional covariates in these models did not alter the effect sizes, and model comparisons using likelihood ratio test were not significant (data not shown). Residuals from linear models were normally distributed. Stepwise regression revealed that OPG$\mathrm{Ab}$ concentration, age, gender and BMI were independently and significantly associated with total hip BMD (g/ $\mathrm{cm}^{2}$ ). In contrast, only age and bisphosphonate use were associated with femoral neck BMD $\left(\mathrm{g} / \mathrm{cm}^{2}\right)$, while age, gender and BMI were associated with spinal BMD $\left(\mathrm{g} / \mathrm{cm}^{2}\right)$.

No associations were observed between positive OPG$\mathrm{Ab}$ status or concentration and markers of disease activity (data not shown). OPG-Ab concentration was associated with OPG ( $\rho=0.17, p=0.046)$ but not with sRANKL. There were no associations between OPG-Ab and CTX/ PINP in the total cohort, nor in the subgroup not receiving bisphosphonates. sRANKL was associated with OPG $(\rho=-0.38, p<0.001)$ and PINP $(\rho=0.19, p=0.032)$, and PINP was associated with CTX $(\rho=0.70, p<0.001)$.
Serum concentrations of OPG, sRANKL, CTX and PINP were not associated with fractures, hip or spine BMD (data not shown).

\section{Discussion}

This cross-sectional study has demonstrated that $8.2 \%$ of patients with established axSpA were positive for OPG-Ab. These autoantibodies were independently associated with reduced hip BMD and a history of self-reported fractures. Adjusted for confounders, regression models showed that patients positive for OPG-Ab had lower total hip BMD by $0.15 \mathrm{~g} / \mathrm{cm}^{2}$ or 1 unit in $T$-score than those who were negative. For each unit increase in OPG-Ab concentration, total hip $T$-score was 0.05 unit lower. Participants positive for OPG-Ab had 24-fold increased likelihood of hip osteopenia and tenfold increased likelihood of reporting a prior fracture.

The prevalence of positive OPG-Ab is similar to that reported in RA $(9.3 \%)$ and coeliac disease $(9.8 \%)$ but is significantly higher than that reported in healthy controls $(1-1.4 \%)[13,14]$. This was unexpected since axSpA is not classically associated with the development of autoantibodies.

Previous studies have demonstrated associations between OPG-Ab and severe osteoporosis and fractures in a patient with coeliac disease and hypothyroidism, as well as increased bone resorption in RA and low BMD in coeliac disease [12-14]. However, this is the first study to show that positive OPG-Ab is associated with reduced hip $\mathrm{BMD}$ in axSpA. The lack of association with spine BMD 
Fig. 2 Scatter plot of anti-OPG antibody concentration against bone mineral density $\left(\mathrm{g} / \mathrm{cm}^{2}\right)$ of total hip, femoral neck and lumbar spine. Lines show least square lines of best fit
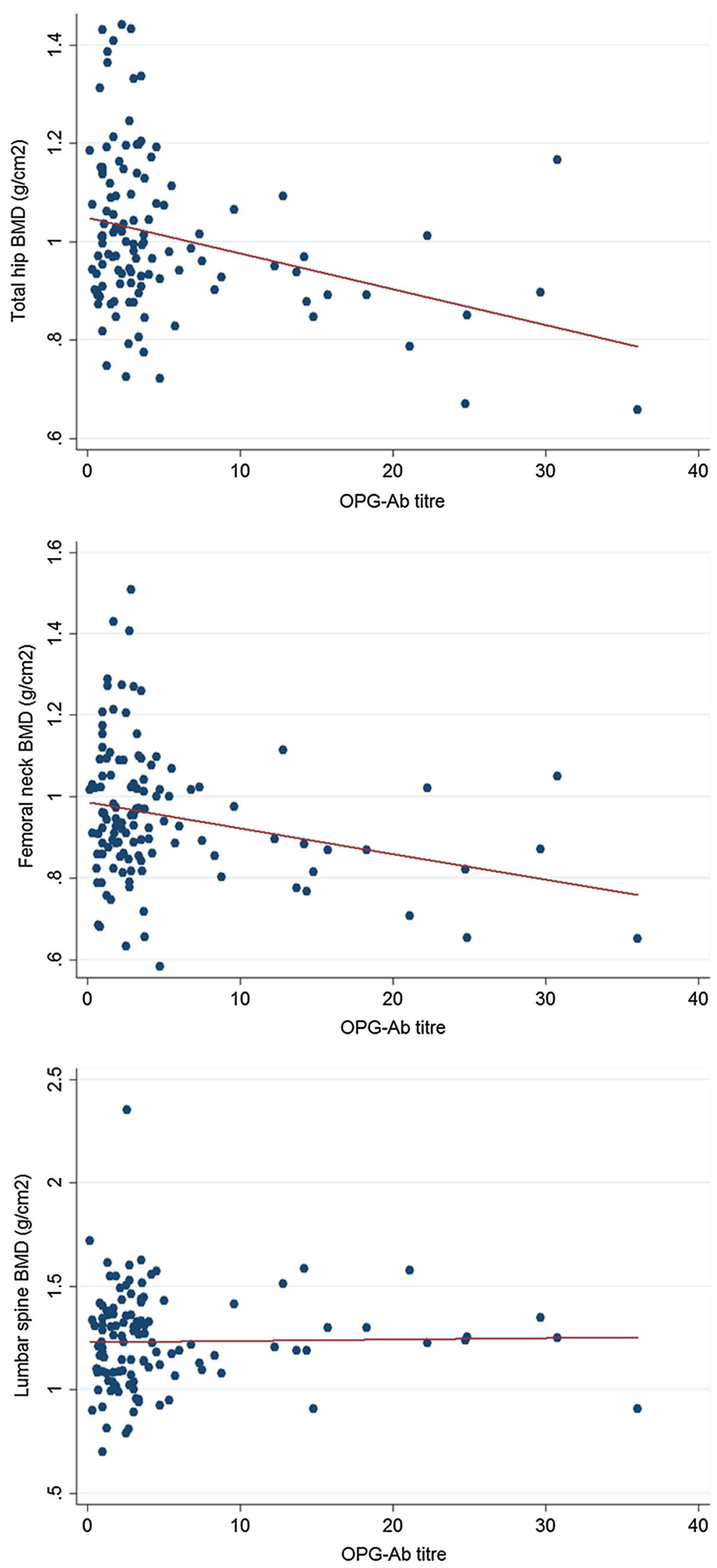
Table 2 Multivariable linear and logistic regression models demonstrating associations between BMD and fractures and OPG-Ab, adjusted for age, gender, disease duration, bisphosphonate use, BASDAI and BMI (age was not included for Z-score models)

\begin{tabular}{|c|c|c|c|c|c|c|}
\hline & \multicolumn{3}{|c|}{ OPG-Ab positivity } & \multicolumn{3}{|c|}{ OPG-Ab concentration } \\
\hline & Effect size & $\begin{array}{l}95 \% \text { confidence } \\
\text { interval }\end{array}$ & $\begin{array}{l}R^{2} / \text { pseudo } \\
R^{2}\end{array}$ & Effect size & $\begin{array}{l}95 \% \text { confidence } \\
\text { interval }\end{array}$ & $\begin{array}{l}R^{2} / \text { pseudo } \\
R^{2}\end{array}$ \\
\hline Total hip BMD $\left(\mathrm{g} / \mathrm{cm}^{2}\right)$ & $\beta=-0.15$ & $-0.25,-0.04$ & 0.26 & $\beta=-0.01$ & $-0.01,-0.00$ & 0.26 \\
\hline Total hip $T$-score & $\beta=-1.06$ & $-1.82,-0.30$ & 0.21 & $\beta=-0.05$ & $-0.09,-0.02$ & 0.22 \\
\hline Total hip Z-score & $\beta=-1.12$ & $-1.88,-0.36$ & 0.11 & $\beta=-0.05$ & $-0.09,-0.02$ & 0.11 \\
\hline Femoral neck BMD $\left(\mathrm{g} / \mathrm{cm}^{2}\right)$ & $\beta=-0.09$ & $-0.20,0.02$ & 0.25 & $\beta=-0.00$ & $-0.01,0.00$ & 0.25 \\
\hline Femoral neck $T$-score & $\beta=-0.72$ & $-1.52,0.07$ & 0.27 & $\beta=-0.03$ & $-0.07,0.00$ & 0.27 \\
\hline Femoral neck Z-score & $\beta=-0.79$ & $-1.58,-0.01$ & 0.11 & $\beta=-0.03$ & $-0.07,0.00$ & 0.10 \\
\hline Lumbar spine $\mathrm{BMD}\left(\mathrm{g} / \mathrm{cm}^{2}\right)$ & $\beta=-0.06$ & $-0.21,0.08$ & 0.27 & $\beta=-0.00$ & $-0.01,0.00$ & 0.27 \\
\hline Lumbar spine $T$-score & $\beta=-0.47$ & $-1.69,0.76$ & 0.23 & $\beta=-0.02$ & $-0.07,0.04$ & 0.23 \\
\hline Lumbar spine $Z$-score & $\beta=-0.73$ & $-2.00,0.55$ & 0.21 & $\beta=-0.02$ & $-0.08,0.04$ & 0.21 \\
\hline Total hip osteopenia $(T$-score $<-1)$ & $\mathrm{OR}_{\mathrm{adj}} 24.2$ & $2.57,228$ & 0.19 & OR $_{\text {adj }} 1.19$ & $1.07,1.31$ & 0.21 \\
\hline Total hip osteoporosis $(T$-score $\leq-2.5)$ & $\mathrm{OR}_{\mathrm{adj}} 45.3$ & $0.22,9521$ & 0.61 & $\mathrm{OR}_{\mathrm{adj}} 1.11$ & $0.92,1.34$ & 0.57 \\
\hline $\begin{array}{l}\text { Femoral neck osteopenia }(T \text { - } \\
\text { score }<-1)\end{array}$ & $\mathrm{OR}_{\mathrm{adj}} 21.3$ & $1.96,231$ & 0.25 & $\mathrm{OR}_{\mathrm{adj}} 1.17$ & $1.05,1.30$ & 0.26 \\
\hline $\begin{array}{l}\text { Femoral neck osteoporosis ( } T \text { - } \\
\text { score } \leq-2.5 \text { ) }\end{array}$ & $\mathrm{OR}_{\text {adj }} 1.41$ & $0.08,26.4$ & 0.55 & $\mathrm{OR}_{\mathrm{adj}} 1.01$ & $0.89,1.13$ & 0.55 \\
\hline History of previous fracture & $\mathrm{OR}_{\mathrm{adj}} 10.5$ & $\mathbf{2 . 0 7}, \mathbf{5 3 . 3}$ & 0.10 & OR $_{\text {adj }} 1.09$ & $1.01,1.17$ & 0.08 \\
\hline
\end{tabular}

Statistically significant models are in bold. Complete data for all covariates were available for 116 in the femoral neck and total hip models. Complete data for all covariates of the fracture model were available for 128

$B M D$ bone mineral density, $O P G-A b$ anti-OPG antibody

may reflect syndesmophytes increasing anteroposterior $\mathrm{BMD}$, even though there may be osteoporosis affecting the trabecular compartment of the vertebrae [1]. Future studies should utilise lateral DXA scans or quantitative CT to investigate the vertebral trabecular compartment BMD in relation to OPG-Ab in axSpA.

Since previous studies have shown that OPG-Abs block the inhibitory effect of OPG on RANKL-induced NFKB signalling in vitro, this provides an explanation for the association between OPG-Ab and bone loss [12, 13]. The findings reported here suggest that these antibodies may also contribute to the pathogenesis of systemic bone loss in some axSpA patients. Nonetheless, we acknowledge that OPG-Abs are likely to be only one of many contributory factors to the pathogenesis of bone loss in this complex disease.

In order to explore the pathophysiological mechanisms by which OPG-Ab were associated with reduced BMD in this population, we studied the relation between biochemical markers of bone resorption and bone formation and OPG-Ab, as well as possible associations between OPG-Ab and levels of OPG and free sRANKL. We were surprised to find a positive association between circulating levels of OPG and OPG-Ab. This has not been observed before [22] but a possible explanation might be that in some axSpA cases, local or systemic release of OPG may trigger an OPG-Ab response. We also found a negative correlation between OPG-Ab and free sRANKL concentrations. This is likely to be due to interference of OPG-Ab with the Biomedica sRANKL assay since this ELISA uses OPG as the capture antigen. It is therefore likely that OPG-Ab binds to the capture antigen, and in doing so blocks its ability to bind to free sRANKL. We found no correlation between OPG-Ab and CTX or PINP concentrations although this may have been due to the fact that one-third of patients with OPG-Ab were on bisphosphonates for the treatment of their osteoporosis. Further studies in untreated patients with axSpA would be warranted to determine if $\mathrm{OPG}-\mathrm{Ab}$ are associated with increased bone turnover as has been reported previously $[12,13]$.

Patients positive for OPG-Ab were older and had longer disease duration. It is known that autoantibody production increases with age. However, associations in this study remained significant after adjusting for both age and disease duration. An association was found between OPG-Ab positivity and increasing disease duration, which was also observed in RA [13]. The reason for this association remains unexplained but we hypothesise that a cycle of chronic inflammation, $\mathrm{B}$ and $\mathrm{T}$-cell proliferation and chronically increased RANKL/OPG concentrations may 
increase the risk of breaking tolerance and promote autoantibody production. Longitudinal studies are required to explore the timing or triggering factor of $\mathrm{OPG}-\mathrm{Ab}$ development during the course of disease. Height was lower in patients positive for OPG-Ab compared with those who were negative. This may have been explained by their older age, but another possibility would be the increased prevalence of vertebral fractures in this group. No associations were demonstrated between OPG-Ab and disease activity or inflammatory markers which suggest that OPG$\mathrm{Ab}$ may be independent of local inflammation in axSpA.

This small cohort had variable exposures to non-biological DMARDs and NSAIDs. While no association with these drug exposures was observed, the study was not adequately powered to explore such associations with OPG-Ab development. The association between TNFi use and OPG-Ab requires further exploration. Patients commenced on TNFi would have higher disease activity and inflammatory burden, which may promote autoantibody formation. Low BMD and fracture history were more prevalent in OPG-Ab-positive patients, which would explain increased bisphosphonate use in this group. A similar trend for increased TNFi and bisphosphonate use was seen in OPG-Ab-positive RA patients [13].

There were several limitations in this study. The association with fractures, although statistically significant, was based on a small number of events which are reflected by the wide confidence intervals. The intervals between measurement of OPG-Ab and BMD were variable. Although the median time between recruitment and DXA scan was 0.2 months, we included BMD results from up to four years before and one year after recruitment. We do not think that this would have substantially altered results since BMD is likely to have been relatively stable in this cohort of patients with well-established axSpA. In this regard, it has previously been found that accelerated bone loss predominantly occurs in early disease [7] and so it is likely that, in this population where median disease duration was 6.4 years, BMD results would have been stable. Another limitation is that the findings were primarily based on male axSpA patients who are more commonly affected by accelerated bone loss and vertebral fractures [8]. Due to the small number of female patients, it was not possible to evaluate the impact of gender on OPG-Ab positivity. Given most patients had established disease, it is important to emphasise that the results cannot be extrapolated to newly diagnosed younger patients who are at relatively higher risk of osteoporotic fractures [7]. A potential limitation was that not all self-reported fractures could be validated but this is unlikely to have resulted in systematic bias since fracture assessments were completed before the measurements for OPG-Ab were performed. It is of interest that the proportion of vertebral fractures in this cohort was lower than that reported in some historical cohorts [2]. This may reflect improved management or may have been due to the fact that lateral spine imaging was only performed in patients with symptoms suggestive of a clinical vertebral fracture. Because of that it is entirely possible that morphometric fractures may have not been detected.

In conclusion, the results of this study raise the possibility that OPG-Ab may play a role in axSpA-associated systemic bone loss in some patients and that these antibodies may be a biomarker for risk of bone loss and fractures. However, a causative role cannot be confirmed by this cross-sectional study. Larger longitudinal studies are needed to establish and quantify the impact of OPG-Ab, their functionality, and to explore the potential of early detection and prevention of OPG-Ab associated osteoporosis.

Acknowledgements We thank research nurses Helen Frankland and Ayren Mediana for recruiting patients. We also thank laboratory scientists Dr Andrew Cross and Jenny Hawkes for technical assistance.

Author Contributions SZ drafted the manuscript with significant contribution from $\mathrm{BH}$. All authors participated in critically revising the manuscript with important intellectual content. All authors contributed to design of the study: Laboratory methodology was designed and/or performed by BH, MRV, PLR, IP, WDF and SHR. Analysis was designed and performed by SZ and NJG. All authors approved the final version of the submitted manuscript.

Funding This study was supported by a National Institute for Health Research (NIHR) collaboration fund awarded to SZ, and by an Arthritis Research UK clinical studentship awarded to BH and SR.

\section{Compliance with Ethical Standards}

Conflict of interest PLR, SHR and WDF have a patent application pending relating to the detection of osteoprotegerin antibodies in the diagnosis of osteoporosis.

Ethical Approval This study received ethical approval from both the Liverpool (14/NW/1320) and Edinburgh sites (10/S1402/33).

Human and Animal Rights and Informed Consent All procedures performed in studies involving human participants were in accordance with the ethical standards of the institutional and/or national research committee and with the 1964 Helsinki declaration and its later amendments or comparable ethical standards. Informed consent: Informed consent was obtained from all individual participants included in the study.

Open Access This article is distributed under the terms of the Creative Commons Attribution 4.0 International License (http://creative commons.org/licenses/by/4.0/), which permits unrestricted use, distribution, and reproduction in any medium, provided you give appropriate credit to the original author(s) and the source, provide a link to the Creative Commons license, and indicate if changes were made.

\section{References}

1. Donnelly S, Doyle DV, Denton A, Rolfe I, McCloskey EV, Spector TD (1994) Bone mineral density and vertebral 
compression fracture rates in ankylosing spondylitis. Ann Rheum Dis 53(2):117-121

2. Ralston SH, Urquhart GD, Brzeski M, Sturrock RD (1990) Prevalence of vertebral compression fractures due to osteoporosis in ankylosing spondylitis. BMJ 300(6724):563-565

3. Will R, Palmer R, Bhalla AK, Ring F, Calin A (1989) Osteoporosis in early ankylosing spondylitis: a primary pathological event? Lancet 2(8678-8679):1483-1485

4. Ghozlani I, Ghazi M, Nouijai A, Mounach A, Rezqi A, Achemlal L, Bezza A, El Maghraoui A (2009) Prevalence and risk factors of osteoporosis and vertebral fractures in patients with ankylosing spondylitis. Bone 44(5):772-776. doi:10.1016/j.bone.2008.12. 028

5. Montala N, Juanola X, Collantes E, Munoz-Gomariz E, Gonzalez C, Gratacos J, Zarco P, Fernandez Sueiro JL, Mulero J, TorreAlonso JC, Batlle E, Carmona L (2011) Prevalence of vertebral fractures by semiautomated morphometry in patients with ankylosing spondylitis. The Journal of Rheumatology 38(5):893-897. doi:10.3899/jrheum.100851

6. Cooper C, Carbone L, Michet CJ, Atkinson EJ, O'Fallon WM, Melton LJ 3rd (1994) Fracture risk in patients with ankylosing spondylitis: a population based study. The Journal of Rheumatology 21(10):1877-1882

7. van der Weijden MA, van der Horst-Bruinsma IE, van Denderen JC, Dijkmans BA, Heymans MW, Lems WF (2012) High frequency of vertebral fractures in early spondylarthropathies. Osteoporos Int 23(6):1683-1690. doi:10.1007/s00198-011-1766$\mathrm{z}$

8. van der Weijden MA, van Denderen JC, Lems WF, Heymans MW, Dijkmans BA, van der Horst-Bruinsma IE (2011) Low bone mineral density is related to male gender and decreased functional capacity in early spondylarthropathies. Clin Rheumatol 30(4):497-503. doi:10.1007/s10067-010-1538-8

9. Kanis JA, Oden A, Johansson H, Borgstrom F, Strom O, McCloskey E (2009) FRAX and its applications to clinical practice. Bone 44(5):734-743. doi:10.1016/j.bone.2009.01.373

10. Wendling D, Cedoz JP, Racadot E, Dumoulin G (2007) Serum IL-17, BMP-7, and bone turnover markers in patients with ankylosing spondylitis. Joint, Bone, Spine 74(3):304-305. doi:10. 1016/j.jbspin.2006.11.005

11. Boyce BF, Xing L (2008) Functions of RANKL/RANK/OPG in bone modeling and remodeling. Arch Biochem Biophys 473(2):139-146. doi:10.1016/j.abb.2008.03.018

12. Riches PL, McRorie E, Fraser WD, Determann C, van't Hof R, Ralston SH (2009) Osteoporosis associated with neutralizing autoantibodies against osteoprotegerin. The New England Journal of Medicine 361(15):1459-1465. doi:10.1056/NEJMoa0810925
13. Hauser B, Riches PL, Gilchrist T, Visconti MR, Wilson JF, Ralston SH (2015) Autoantibodies to osteoprotegerin are associated with increased bone resorption in rheumatoid arthritis. Ann Rheum Dis 74(8):1631-1632. doi:10.1136/annrheumdis-2014207219

14. Real A, Gilbert N, Hauser B, Kennedy N, Shand A, Gillett H, Gillett P, Goddard C, Cebolla A, Sousa C, Fraser WD, Satsangi J, Ralston SH, Riches PL (2015) Characterisation of osteoprotegerin autoantibodies in coeliac disease. Calcif Tissue Int 97(2):125-133. doi:10.1007/s00223-015-0023-4

15. van der Linden S, Valkenburg HA, Cats A (1984) Evaluation of diagnostic criteria for ankylosing spondylitis. A proposal for modification of the New York criteria. Arthritis Rheum 27(4):361-368

16. Rudwaleit M, Jurik AG, Hermann KG, Landewe R, van der Heijde D, Baraliakos X, Marzo-Ortega H, Ostergaard M, Braun J, Sieper J (2009) Defining active sacroiliitis on magnetic resonance imaging (MRI) for classification of axial spondyloarthritis: a consensual approach by the ASAS/OMERACT MRI group. Ann Rheum Dis 68(10):1520-1527. doi:10.1136/ard.2009.110767

17. Hui SL, Gao S, Zhou XH, Johnston CC Jr, Lu Y, Gluer CC, Grampp S, Genant H (1997) Universal standardization of bone density measurements: a method with optimal properties for calibration among several instruments. J Bone Miner Res 12(9):1463-1470. doi:10.1359/jbmr.1997.12.9.1463

18. Lu Y, Fuerst T, Hui S, Genant HK (2001) Standardization of bone mineral density at femoral neck, trochanter and Ward's triangle. Osteoporos Int 12(6):438-444. doi:10.1007/ s001980170087

19. Allali F, Breban M, Porcher R, Maillefert JF, Dougados M, Roux C (2003) Increase in bone mineral density of patients with spondyloarthropathy treated with anti-tumour necrosis factor alpha. Ann Rheum Dis 62(4):347-349

20. Sutula CL, Gillett JM, Morrissey SM (1986) Ramsdell DC interpreting ELISA data and establishing the positive-negative threshold. Plant Dis 70:722. doi:10.1094/PD-70-722

21. Stern A, Laughlin GA, Bergstrom J, Barrett-Connor E (2007) The sex-specific association of serum osteoprotegerin and receptor activator of nuclear factor kappaB legend with bone mineral density in older adults: the Rancho Bernardo study. Eur J Endocrinol 156(5):555-562. doi:10.1530/EJE-06-0753

22. Hauser B, Riches PL, Gilchrist T, Visconti MR, Ralston SH (2016) Correlation of RANKL, OPG and OPG antibodies in rheumatic diseases. Ann Rheum Dis 75(Suppl 1):A79-A80 